\title{
Evaluation of ZigBee Based Wireless Sensor Network with Static Sink and Random Sink Mobility
}

\author{
Abdul Aziz, M. Ali Qureshi, M. Umair Soorage, M. Noman Kashif, and M. Arsalan Hafeez, Member, \\ IACSIT
}

\begin{abstract}
In this paper we investigate performance of a ZigBee based wireless sensor network in two scenarios one with a static sink and other with random sink mobility through extensive ns2-based simulations for various topologies (star, tree and mesh). Wireless Sensor Networks (WSNs) can be considered as a glut of distributed sensor nodes which are able to sense a physical process or environmental parameters and also capable to transfer that information to a predefined sink node through some intermediate nodes. If sink remains static at a fixed position then neighboring nodes of the sink consume their energy more rapidly as compared to other nodes which are far away from the sink because they have to forward all the traffic of the farther away nodes to the sink that leads to complete isolation of sink in a less time and hence network performance is degraded due to non-uniform consumption of energy. We introduce a simple random mobility scheme in which sink moves randomly through whole network. We also investigate end-to-end delay of packets and throughput for identical network conditions in both the cases. The main aim of this work is to evaluate, through simulations, the impact of random sink mobility in a ZigBee/IEEE 802.15.4 based wireless sensor network.
\end{abstract}

Index Terms-Random mobility, WSN, ZigBee, IEEE 802.15.4, sink.

\section{INTRODUCTION}

Wireless Sensor Networks (WSN) are independent self-configuring networks. These are widely used to monitor and control various parameters of a process in an industry, office home, or any other remote outdoor environment. WSNs may be composed of thousands of little and low energy nodes, which are able to communicate with each other to collectively sense various environment parameters. Numerous battery replacements are not possible because number of nodes is very large. Therefore network lifetime is the main issue for designing algorithms, communication protocols and hardware. Hence extension in network lifetime and effective utilization of energy are the most important research issues regarding wireless sensor networks [1].

Data collection is a basic task of WSN. It aims to collect sensor readings from sensory field at pre-defined sinks for analysis and processing. Recently, sink mobility has been exploited to reduce and balance energy expenditure among sensors. The nodes which are in the neighborhood of the sink will utilize their energy more rapidly because all the data sent by the far away nodes will be gathered by the neighboring

Manuscript received June 12, 2012; revised July 26, 2012.

M. A. and A. Aziz are with Islamia University of Bahawalpur, Pakistan (e-mail: ali.qureshi@iub.edu.pk; abdul.aziz@ iub.edu.pk). nodes and then forwarded to the sink. To balance the energy consumption in a network a mobile sink can be very helpful because due to movement of sink the neighboring nodes of the sink consciously changes and hence improvement in the network lifetime. Mobile sinks reduce end to end delay, increases success rate and decreases energy dissipation in wireless sensor networks [2].

\section{RelATED RESEARCH WORK}

Studies on sink mobility has been initiated recently and discussed in [2]-[5]. Most research studies in [2], [3] are related to theoretical aspects of WSNs that are not much helpful to implement real time routing protocols. One problem of these studies is that no real life WSNs, like ZigBee based WSN has been considered. Utilization of mobile sinks in a ZigBee based WSN has been considered in [4], [5]. But these studies did not include random mobility.

Sink mobility to increase lifetime of a WSN has been investigated in several papers [6], [7]. It is explored in [6], that by controlling the sink movements it is possible to obtain significant lifetime improvements.

Considering mobility to improve network performance has been extensively discussed for general ad hoc networks in different perspectives [8], [9]. The main intention of these studies is to transmit messages in disconnected ad hoc networks and to improve network throughput.

\section{ZIGBEE AND IEEE 802.15.4}

ZigBee is a worldwide standard communication protocol designed by the team working under IEEE 802.15 working group-4. ZigBee is the low data rate WPAN standard and has detailed specifications for low data rate nodes, its power consumption is very low and are thus long battery life is possible as compared to other standards like IrDA and Bluetooth because these standards have been designed for high data rate applications such as video, voice and other multimedia applications [10].

ZigBee devices transmit data in range of 10-75 meters, that depends on the RF environment and the transmitter power consumed to transmit data, it works in the unlicensed band worldwide ( $868 \mathrm{MHz}, 915 \mathrm{MHz}$ or $2.4 \mathrm{GHz})$. Its defined data rates are $20 \mathrm{kbps}$ at $868 \mathrm{MHz}, 40 \mathrm{kbps}$ at $915 \mathrm{MHz}$ and $250 \mathrm{kbps}$ at $2.4 \mathrm{GHz}$ [11].

IEEE and ZigBee Alliance have been working together to define all the protocols for IEEE 802.15.4/ZigBee. IEEE has the responsibility to define protocols for the two lower layers, MAC and physical. While ZigBee Alliance focuses on the 
upper layers (from network to the application layer). IEEE 802.15.4 now has all the detailed specifications physical and MAC layers for developing different types of topologies like star, mesh, and tree. Various network routing protocols are designed for low power conservation, and to ensure low end-to-end delay through guaranteed time slots (GTS) [12].

\section{A. Network Topologies}

ZigBee networks are a combination of three types of devices [13]. These devices are:

- ZigBee coordinator

- ZigBee router

- ZigBee end device

ZigBee coordinator - There can be only one coordinator for each ZigBee network. This node is liable for initializing the network, selecting the suitable channel and allowing other devices to connect to its network.

ZigBee Router - A router is liable to pass on messages in a network, and is also able to connect to another router, or an end device. Router functions are only used in a tree or mesh topology.

ZigBee End Device - These nodes are only used to talk either a router or a coordinator. An end device connected to the network through either a router, or directly to the coordinator.

There are three different types of topologies possible for a ZigBee network [14].

- Star Topology

- Tree Topology

- Mesh Topology

1) Star topology

In this topology, a coordinator is encircled by a group of either end devices or routers. The main advantage of this topology is its simplicity, but the main disadvantage is that if the coordinator stops working then the whole network will become unavailable because all the traffic must travel through the coordinator.

\section{2) Tree topology}

In a tree topology, a coordinator lies at the root of the tree. The coordinator can now be connected to either routers or end devices and the router can be connected to more child nodes. But the end device can not be connected to more child nodes. In this topology we have only one possible path for a message to travel from source to destination, this type of topology is also not reliable to some extent. If a router fails, then all of that router's children are disconnected from rest of the network.

\section{3) Mesh topology}

A mesh topology is the most reliable topology because a message can take multiple paths from source to destination. If a router fails then due to ZigBee's self healing mechanism the message will be transferred through alternate path.

\section{B. Random Sink Mobility}

Random Sink mobility means that the sink move randomly through the entire network. Random mobility is independent of the network topology, data tributaries and residual energy of the nodes (Router, End Device and Sink). Sink randomly selects the distance and route of a section in its pathway. It also randomly selects the time to move on the single segment and selects the pause times between movements along diverse segments [14].
The problem of sink neighborhood can be solved by using Random sink mobility. Energy expenditure among nodes of all parts of the network will become more uniform because neighborhood of sink changes randomly due to random movement of sink. That ultimately improves the network lifetime. There is no overhead for routing in case of random mobility because the selection of sink's route does not depend on any parameters of the network [14].

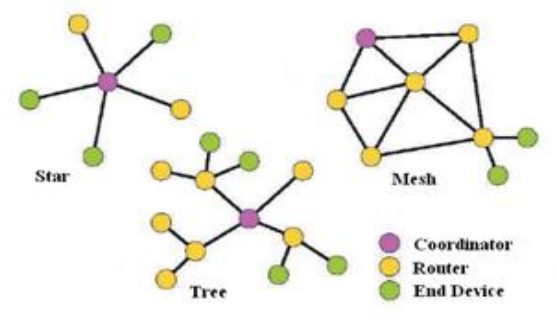

Fig. 1. Network topologies

\section{Simulation Results}

In order to evaluate the performance of ZigBee wireless sensor networks for static sink and random mobility of sink, we have conducted extensive ns2-based simulations for the three topologies (star, tree and mesh) in which we did analysis of the three parameters (consumed energy, end-to-end delay and throughput ) of the nodes. In first part the simulation results for a static sink are given in which first of all the results for average energy consumption for network nodes in case of star topology (Fig-2), tree topology (Fig-3) and mesh topology (Fig-4) are shown then results of end-to-end delay are shown in Fig-5 to Fig-7 and in the last results for average throughput are shown in Fig-8 to Fig-10. Similarly in the second part, simulation results for random mobility of sink are shown in Fig 11 to Fig-19. Simulation parameters used in both the cases for star, tree and mesh topologies are given in Table I. All simulations are performed in identical network conditions with same number of nodes; only in star topology router nodes are not available because there is no role of routers in star topology.

TABLE I: SIMULATION PARAMETERS

\begin{tabular}{ll}
\hline \hline Parameter & Value \\
\hline Operating Frequency & $2.4 \mathrm{GHz}$ (Worldwide ISM band) \\
Channel Banwidth & $250 \mathrm{kbps}$ \\
Simulation Area $\left(\mathbf{L}^{*} \mathbf{W}\right)$ & $500 \mathrm{~m} \mathrm{x} 500 \mathrm{~m}$ \\
Simulation Time & 60 minutes (3600 seconds) \\
ZigBee Routers & 08 (Nill in Star Toplogy) \\
ZigBee End Devices & 12 \\
ZigBee Coordinator & 01 \\
Tx Power (Router, End Device and & $0.05 \mathrm{w}$ \\
Sink) & \\
Initial Energy & $1600 \mathrm{mAH}$ \\
Power Supply & $2 \mathrm{AA} \mathrm{Batteries} \mathrm{(3V)}$ \\
Packet Size & $1024 \mathrm{Bytes}$ \\
Current Drawn in Tx Mode & $17.4 \mathrm{~mA}$ \\
Current Drawn in Rx Mode & $27.7 \mathrm{~mA}$ \\
Current Drawn in Idle Mode & $35 \mu \mathrm{A}$ \\
Current Drawn in Sleep Mode & $16 \mu \mathrm{A}$ \\
\hline \hline
\end{tabular}




\section{A. Simulation Results for Static Sink}

\section{1) Energy consumption for static sink}

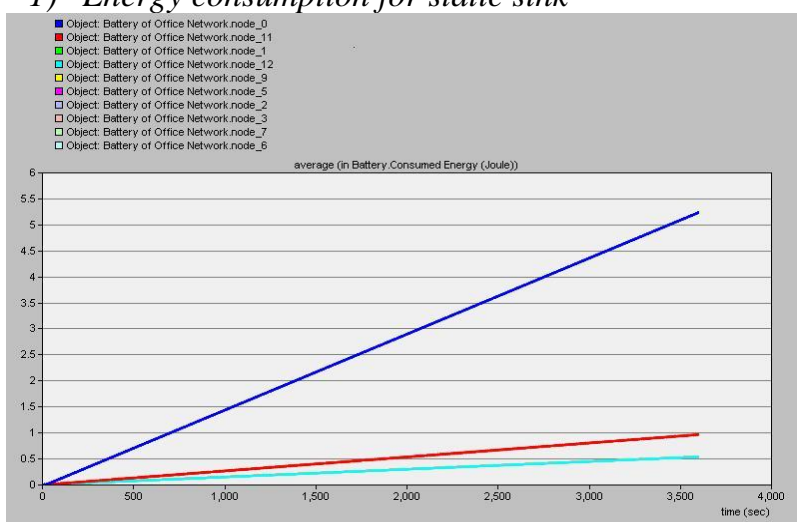

Fig. 2. Average energy expenditure in star topology

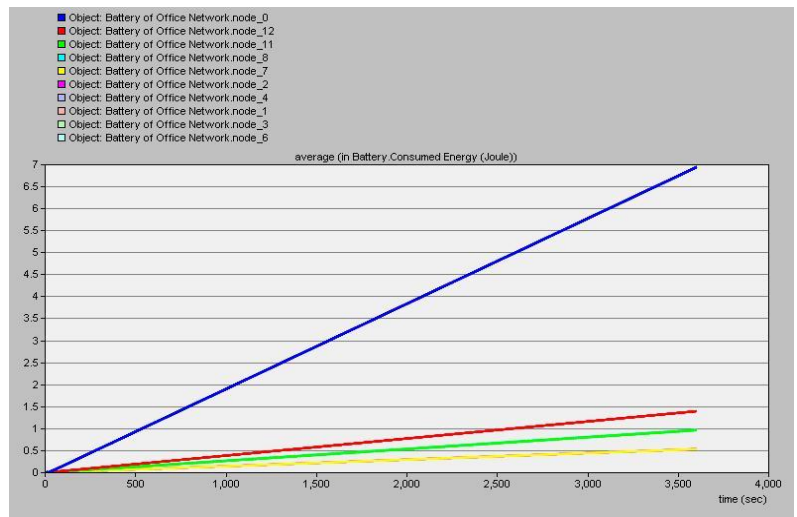

Fig. 3. Average energy expenditure in tree topology

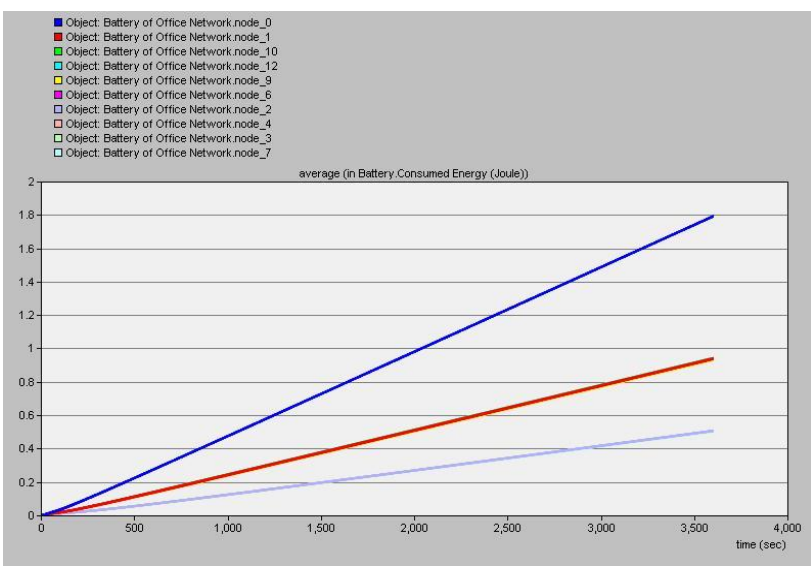

Fig. 4. Average energy expenditure in mesh topology

\section{2) End-to-end delay for static sink}

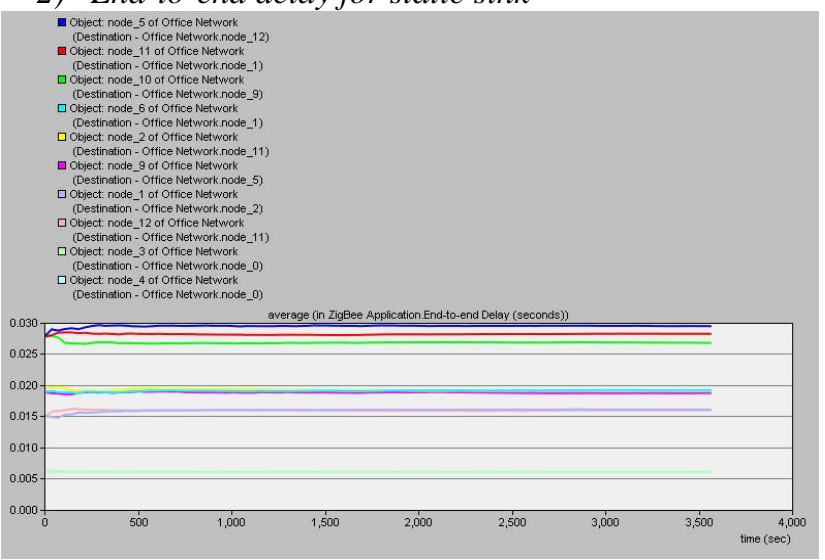

Fig. 5. End-to-end delay in star topology

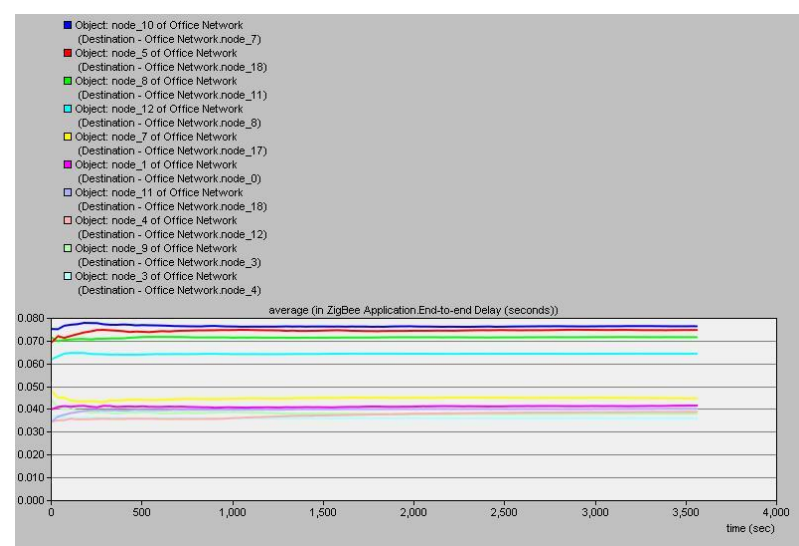

Fig. 6. End-to-end delay in tree topology

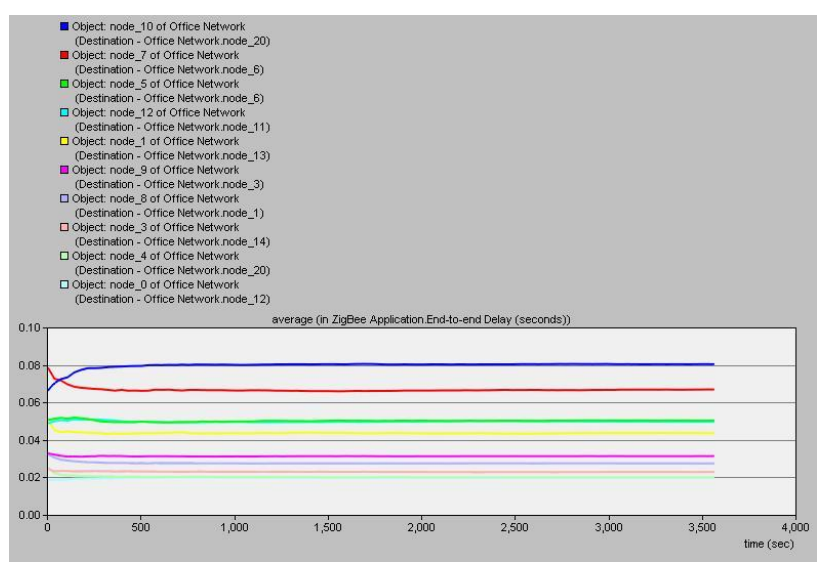

Fig. 7. End-to-end delay in mesh topology

3) Throughput for static sink

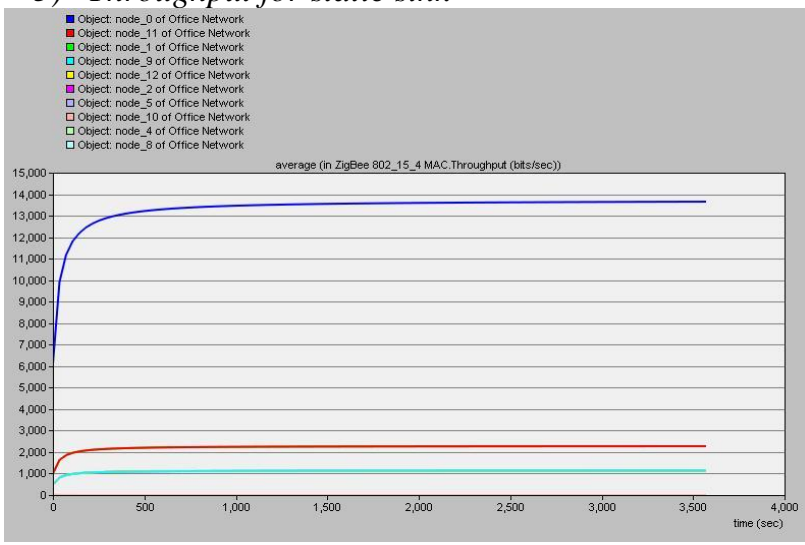

Fig. 8. Throughput in star topology

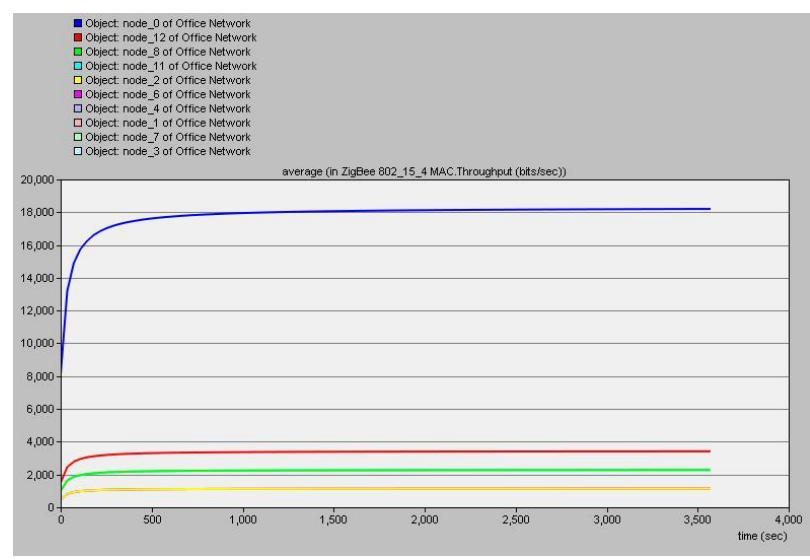

Fig. 9. Throughput in tree topology 


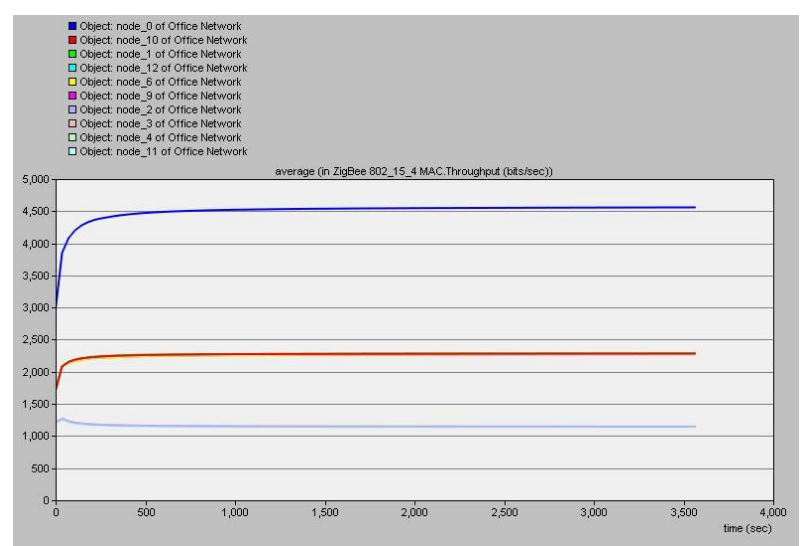

Fig. 10. Average throughput in mesh topology

B. Simulation Results for Random Mobility of Sink

1) Energy consumption for random sink mobility

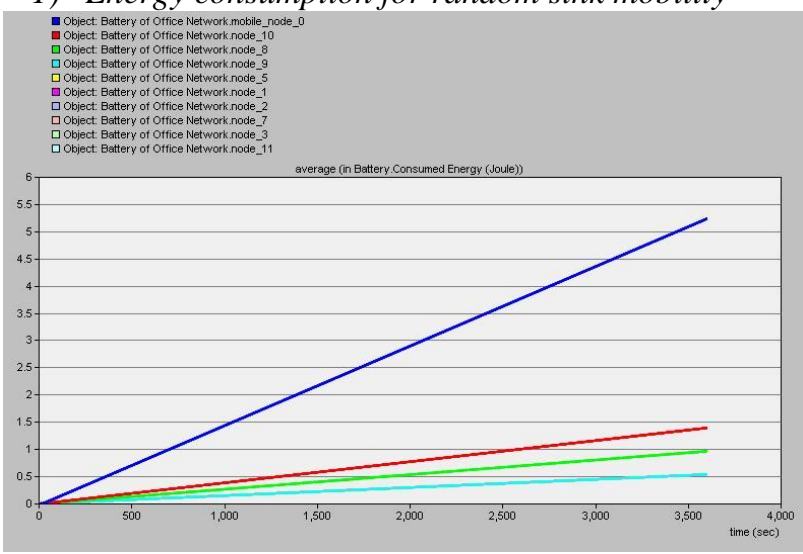

Fig. 11. Average energy expenditure in star topology

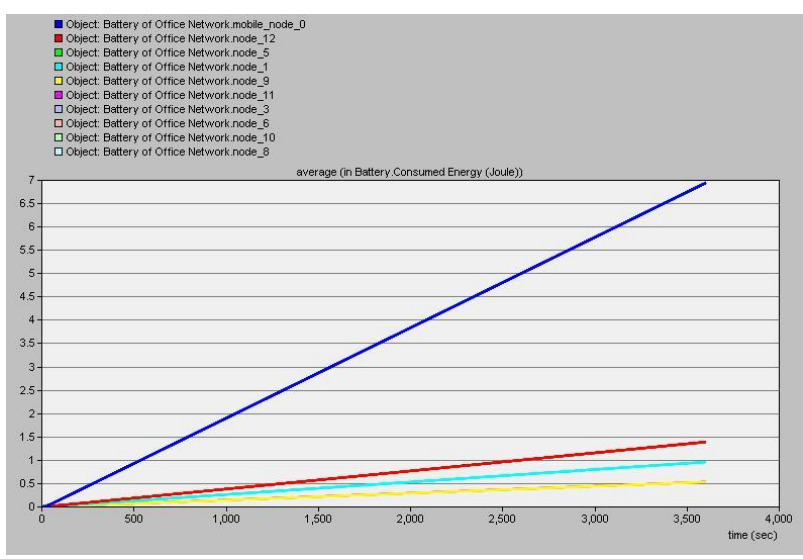

Fig. 12. Average energy expenditure in tree topology

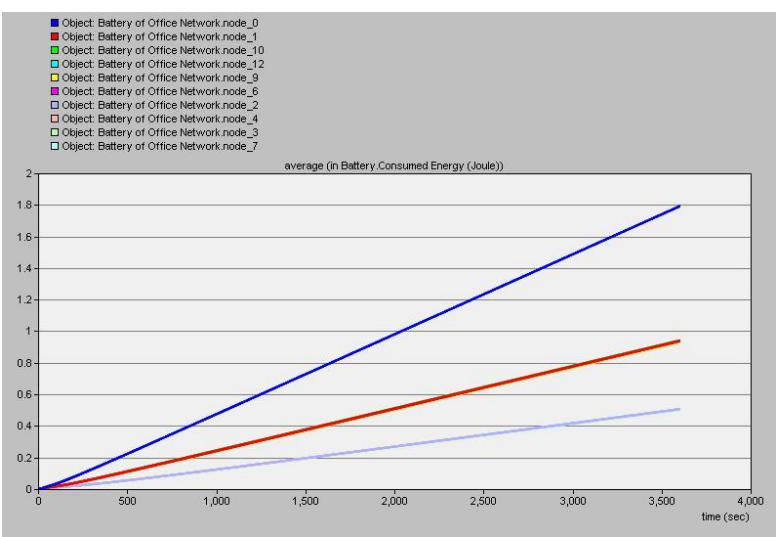

Fig. 13. Average energy expenditure in mesh topology

\section{2) End-to-end delay for random sink mobility}

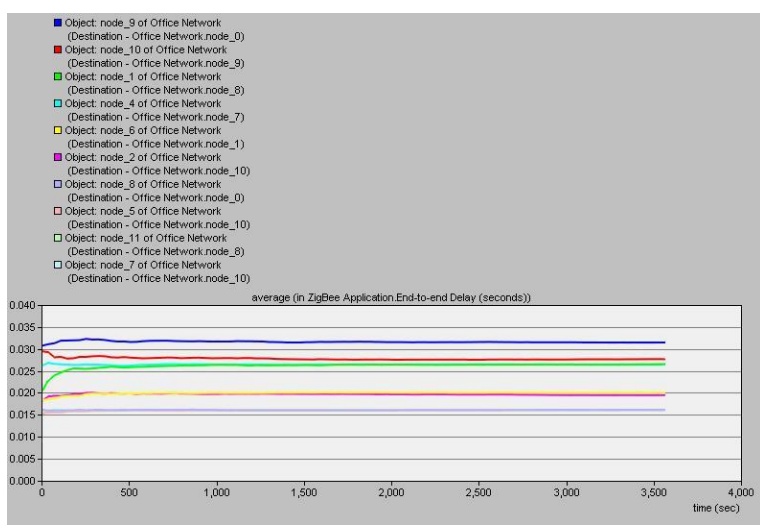

Fig. 14. End-to-end delay in star topology

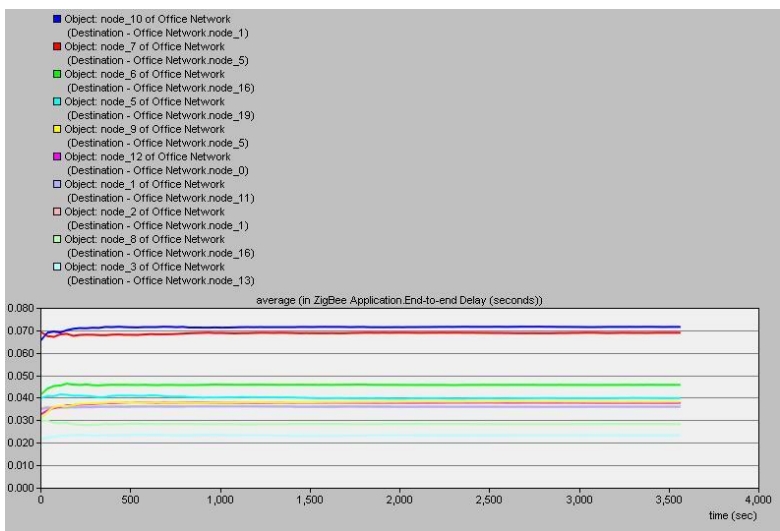

Fig. 15. End-to-end delay in tree topology

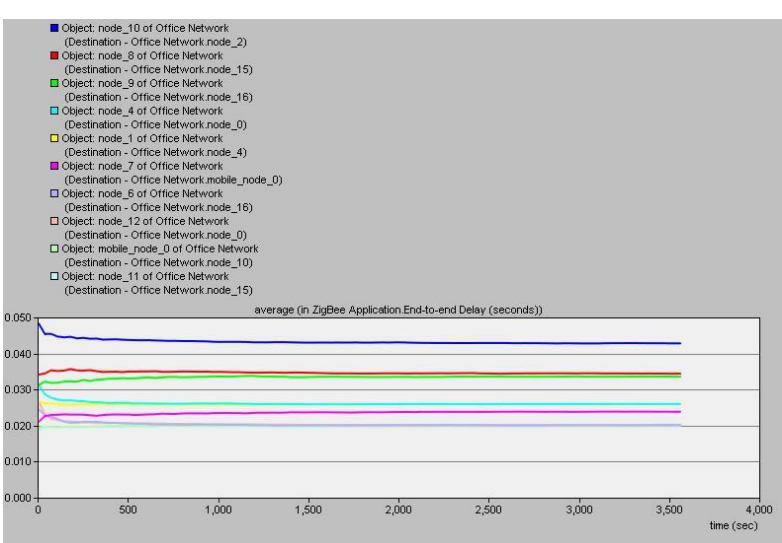

Fig. 16. End-to-end delay in mesh topology

\section{3) Throughput for random sink mobility}

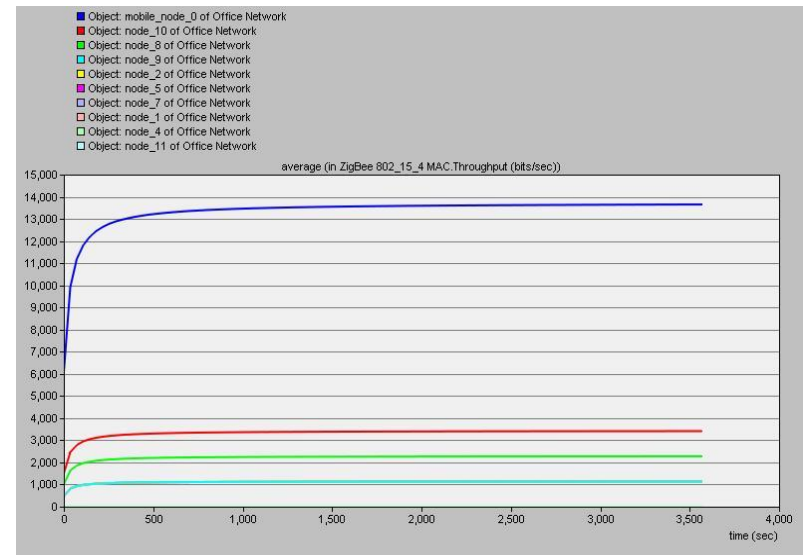

Fig. 17. Throughput in star topology 


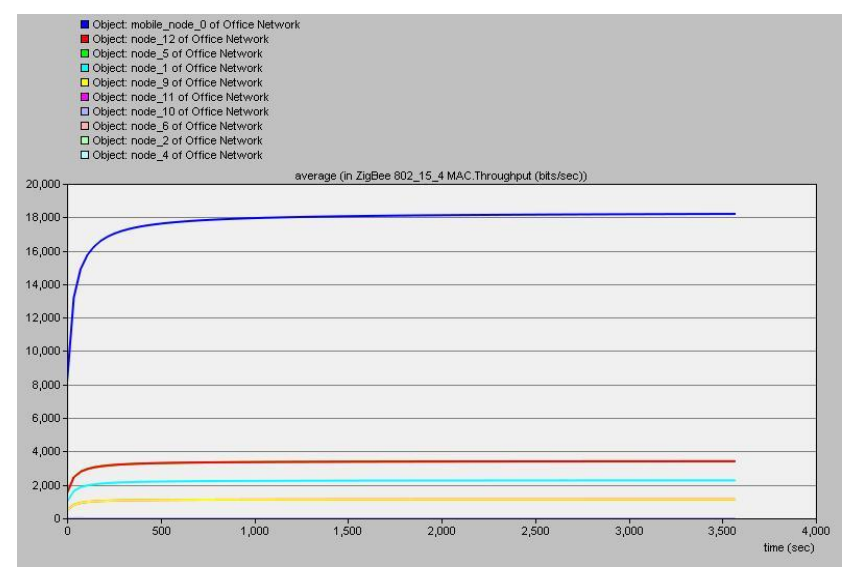

Fig. 18. Throughput in tree topology

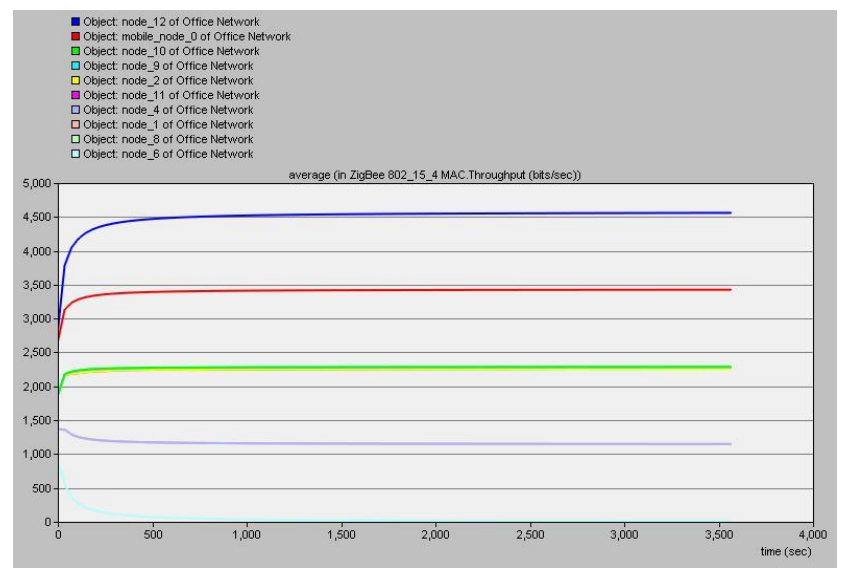

Fig. 19. Throughput in mesh topology

\section{CONCLUSION}

In our study, we compared the impact of random sink mobility and static sink on performance of ZigBee based wireless sensor Network. The performances in both the cases are compared on the basis of average energy consumption, end-to-end delay and throughput. It can be predicted from the simulation results that random sink mobility will provide much better performance as compared to that of static sink, particularly in case of average energy expenditure and end-to-end delay. It is also evident from the results that mesh topology is more energy efficient as compared both topologies and star topology is more efficient as compared to the other topologies in terms of end-to-end delay. Throughput is also significant parameter in the performance of wireless sensor networks; we also observed from the results that tree topology is more efficient as compared to both the topologies in case of throughput.

\section{ACKNOWLEDGMENT}

We would like to thank our respected teachers and colleagues for their guidance and ever present support. We are very grateful to the Institution of Engineers Pakistan (IEP) for providing us support.

We are also very much thankful to Dr. Muhammad Mukhtar, the Vice Chancellor of the university, and Professor Jan Muhammad Keerio, the Principal of UCET, for their efforts to facilitate and provide us most suitable environment for research and development.

\section{REFERENCES}

[1] M. Kohvakka, M. Kuorilehto, M. Hnnikinen, and T. D. Hmlinen, "Performance analysis of IEEE 802.15.4 and ZigBee for large-scale wireless sensor network applications," in Proc. PE-WASUN, Terromolinos, Spain, 2006, pp. 48 - 57.

[2] S. Hashish and A. Karmouch, "Deployment-based solution for prolonging network lifetime in sensor networks," in Proceedings of WSAN'08, 2008.

[3] X. Li, A. Nayak, and I. Stojmenovic, "Sink Mobility in Wireless Sensor Networks," Wireless Sensor and Actuator Networks: Algorithms and Protocols for Scalable Coordination and Data Communication, 2010.

[4] C. Chen and J. Ma, "Simulation study of AODV performance over IEEE 802.15.4 MAC in WSN with mobile sinks," in Proceedings of 21st International Conference on Advanced Information Networking and Applications Workshops, vol. 2, May 2007.

[5] M. Shakya, J. Zhang, P. Zhang, and M. Lampe, "Design and optimization of wireless sensor network with mobile gateway," in Proceedings of 21st International Conference on Advanced Information Networking and Applications Workshops, May 2007.

[6] S. Basagni, A. Carosi, E. Melachrinoudis, C. Petrioli, and Z. M. Wang, "Controlled sink mobility for prolonging wireless sensor networks lifetime," Wireless Networks, vol. 14, no. 6, Dec. 2008.

[7] A. T. Campbell, S. B. Eisenman, N. D. Lane, E. Miluzzo, and R. Peterson, "People-Centric Urban Sensing," In Proc. of Second ACM/IEEE Annual International Wireless Internet Conference (WICON 2006), Boston, USA, 2006.

[8] I. Chatzigiannakis, S. Nikoletseas, and P. Spirakis, "An efficient communication strategy for ad-hoc mobile networks," in Proceedings of the Twentieth Annual ACM Symposium on Principles of Distributed Computing, PODC 2001, Newport, RI, August 26-29 2001, pp. 320-332.

[9] M. Grossglauser and D. N. C. Tse, "Mobility increases the capacity of ad-hoc wireless networks," IEEE/ACM Transactions on Networking, vol. 10, no. 4, pp. 477-486, August 2002.

[10] S. S. R. Ahamed, "The Role of Zigbee Technology in Future Data Communication System," Journal of Theoretical and Applied Information Technology, pp. 129-135, 2005.

[11] Z. Alliance, "ZigBee specification v1.0," June 2005.

[12] S. C. Ergen, "IEEE 802.15.4 Summary," Technical Report, Advanced Technology Lab of National Semiconductor, August 2004.

[13] S. Leung, W. Gomez, J. J. Kim, "ZigBee Mesh Network Simulation using Opnet and Study of Routing Selection," Communication Networks, Final Project, Spring 2009.

[14] D. Stevanovic, N. Vlajic, "Performance of IEEE 802.15.4 in wireless sensor networks with a mobile sink implementing various mobility strategies," LCN, pp.680-688, 2008. 